\title{
Investigations of Resorcinol under High Pressure
}

\author{
M. TYDDA* AND B. JASIŃSKA \\ Department of Nuclear Methods, Institute of Physics, M. Curie-Skłodowska University \\ pl. M. Curie-Skłodowskiej 1, 20-031 Lublin, Poland
}

\begin{abstract}
The investigation of intermolecular free volumes in resorcinol was conducted under high pressure, hydraulic pressure and gas (argon or nitrogen) atmosphere. The sizes of free volumes were estimated basing on the mean lifetime of ortho-positronium (o-Ps). In the first case, a monotonous change of lifetime and intensity was observed for the o-Ps component while the range of pressure was 0-477 $\mathrm{MPa}$. On the other case, the samples were put into the pressure chamber filled with argon or nitrogen in the range of pressure from 0 to $450 \mathrm{MPa}$. When the pressure chamber was filled with a gas, the appearance of an additional o-Ps component has been found. In the nitrogen atmosphere, the material crushed when the gas pressure reached about $200 \mathrm{MPa}$, and the mean lifetime of the longest $o$-Ps component increased.
\end{abstract}

DOI: 10.12693/APhysPolA.125.812

PACS: 78.70.Bj, 36.10.Dr, 61.66.Hq

\section{Introduction}

Positron annihilation lifetime spectroscopy (PALS) is a method based on positron behaviour in the medium. In materials like molecular crystals, polymers and porous media free volumes are present. When the void size is large enough, a bound state of positron with an electron of the medium can be created. It is called positronium $(\mathrm{Ps})$ and is trapped in the void. Positronium exists in two sub states: a short lived, singlet state called para-positronium ( $p$ - Ps $)$ and a long-lived, triplet state called ortho-positronium (o-Ps). In vacuum, these substates have their mean lifetimes determined as $\tau_{p-\mathrm{Ps}}=125 \mathrm{ps}$ and $\tau_{o-\mathrm{Ps}}=142 \mathrm{~ns}$ [1]. In the condensed matter, the $o$-Ps lifetime value can be shortened by the pick-off process. The relation between the free volume size (considered as spherical) and the $o$-Ps mean lifetime value is described by the Tao-Eldrup model $[2,3]$.

An additional parameter obtained from the analysis of the PAL spectra is the relative intensity of all their components, which provides information about the fraction of positrons annihilating in all processes (i.e. the free annihilation of positrons in the material, $p$-Ps and $o$-Ps components).

Intensity of the $o$-Ps components may be affected by numerous factors such as the presence of active groups, radicals, temperature etc. Thus the interpretation of this parameter may not be straightforward.

The aim of our investigations was to compare differences in the o-Ps behaviour in resorcinol under a high pressure of gas atmospheres (argon or nitrogen) and without gas access to the sample.

\section{Experimental}

The investigated material was resorcinol (benzene-1,3-diol) (Fig. 1), one of the three isomers from a group

\footnotetext{
* corresponding author; e-mail: maciej.tydda@poczta.umcs . lublin.pl
}

known as benzenediols which are composed of benzene ring and two hydroxyl groups (the other two isomers are catechol (benzene-1,2-diol) and hydroquinone (benzene$-1,4$-diol)).

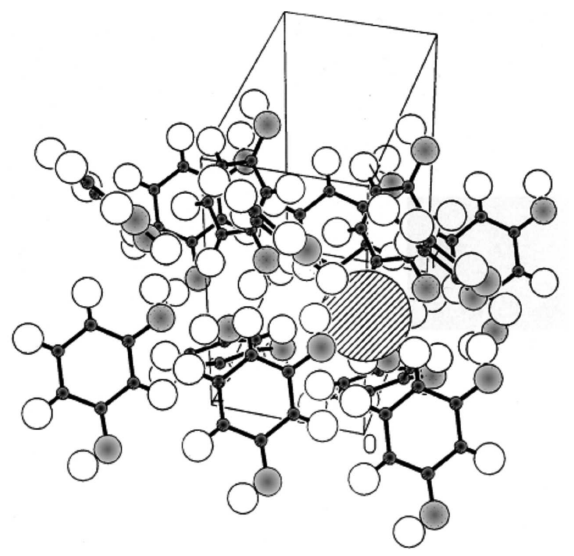

Fig. 1. Crystallographic structure of resorcinol in $\alpha$ phase. Dashed circle shows free volume in the structure. Atoms are drawn by their atomic radii; carbon atoms are not drawn in scale but as points to enhance clarity of the picture. Drawing taken from [5].

In order to collect the positron lifetime spectra a fast-slow spectrometer with a time base of $50 \mathrm{~ns}$ and $\mathrm{BaF}_{2}$ scintillators was used. The time resolution (FWHM) was 0.24 ns. The total number of counts per spectrum was about $3.5 \times 10^{6}$ and it was collected over the time of $8 \mathrm{~h}$. All measurements were conducted at room temperature.

At room temperature resorcinol is a solid and can exist in two crystalline forms $(\alpha, \beta)$ [4]. In this experiment, the more common $\alpha$ phase was investigated. The free volumes inside the crystalline structure of resorcinol can be approximated by a sphere with a diameter of $0.3 \mathrm{~nm}$ [5], as shown in Fig. 1. Previous studies [4] indicate that $o$-Ps lifetime value for this size should be about $0.958 \mathrm{~ns}$, while the values obtained from our experiment is $0.935(6)$ ns which is very close to one calculated 
from crystallographic data, especially when taking into account that the void is only approximated by a sphere.



Fig. 2. Chambers used in experiments: (a) with hydraulic press, (b) gas compressor. Symbols denote: dashed lines - sample; white rectangle - positron source; thick grey arrow (a) - pressure application direction; grey arrows (b) - gas flow direction.

Two types of experiments were performed. In the first one, the samples were placed in a hydraulic press (Fig. 2a). The range of the measurements covered pressures $0-477 \mathrm{MPa}$ in 15 steps. In the analysis of spectra measured in this experiment three exponentials were assumed: a free annihilation of positrons in the sample, $p$-Ps decay and an o-Ps component. The para-positronium lifetime was fixed at $125 \mathrm{ps}$. The dependence of the lifetime of the $o$-Ps component on pressure is found nonlinear and can be approximated by a quadratic function (Fig. 3).
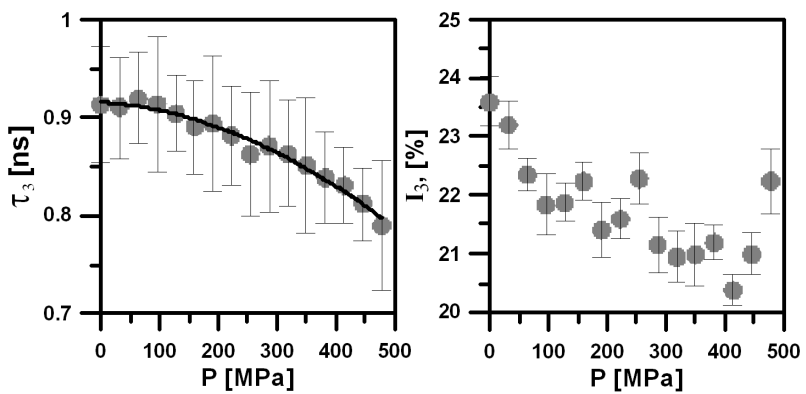

Fig. 3. o-Ps lifetime and intensity data for resorcinol under mechanical pressure. The line shows the second degree polynomial approximating the experimental points.

In the second version of the experiment, the gas compressor (Unipress U11) was used in order to generate high pressure atmospheres (Fig. 2b). Two gases were used during those measurements: nitrogen and argon.

The samples were placed in the chamber of compressor and PAL spectra were registered in the range of pressures of $0-450 \mathrm{MPa}$ in $30 \mathrm{MPa}$ steps. In this version of the experiment, spectra were decomposed into four components (as previous processing with three components was not sufficient): $p$-Ps (lifetime was fixed as previously at $125 \mathrm{ps}$ ), free annihilation of positrons in the sample, and the other two components were ascribed to o-Ps annihilation. The analysis of all these lifetime spectra analysis was conducted with the LT program [6].

\section{Results and discussion}

The experiment without gas access to the sample was carried out as a reference for the next experiment where a sample was exposed to gas atmosphere. The results obtained are shown in Fig. 3.

The $o$-Ps mean lifetime value decreases throughout the pressure range from about $0.9 \mathrm{~ns}$ to $0.8 \mathrm{~ns}$ and as for the intensity, it goes from $24 \%$ to $21 \%$. This indicates that free volume size decreases monotonously in the whole range of applied pressure; the intensity behaves similarly.

In the second version of the experiment two gases were used: argon (which has atomic radius of $0.71 \AA[7])$ and nitrogen (which has atomic radius equal to $0.65 \AA$ [7], and in the molecular form can be described as a cylinder of length $2.6 \AA$ and base diameter of $1.3 \AA$ ). It was expected that these gases would interact with the material and penetrate the free volumes in different ways because of the differences in the shapes of their gas molecules.
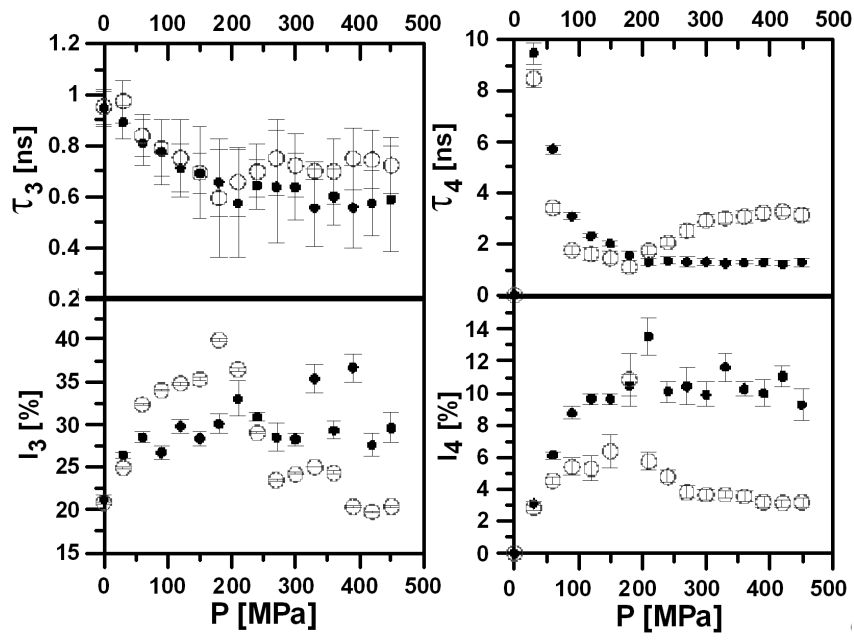

Fig. 4. The lifetime values and intensity of o-Ps components in resorcinol as a function of: argon pressure (filled circles) and nitrogen (empty circles).

In Fig. 4 the lifetimes and intensities of the $o$-Ps components in both argon and nitrogen atmospheres are presented. As it was mentioned above, the second $o-\mathrm{Ps}$ component $\left(\tau_{4}\right)$ is ascribed to gas presence in the chamber. Because of an unexpected course of the lifetime dependence on gas pressure, more than one method of spectra analysis was attempted [8]. Based on the obtained results we found that the analysis with two free o-Ps components gives the most accurate insight into the physical phenomenon.

The lifetime dependence of the shorter $o$-Ps component on pressure (with gas presence) is different than one observed in the measurements without gas access. In the 
range of 0-200 $\mathrm{MPa}$, the lifetime values linearly decrease down to $0.6 \mathrm{~ns}$ at $210 \mathrm{MPa}$. This lifetime value is beneath the lower range for existence of $o-\mathrm{Ps}$.

Above $210 \mathrm{MPa}$ different lifetime values are observed for both gases. When the chamber with the sample is filled with argon, the shortest $o$-Ps component stabilizes at the mentioned level of $0.6 \mathrm{~ns}$. When the sample is placed in a nitrogen atmosphere, the lifetime value raises to $0.7 \mathrm{~ns}$ and stabilizes afterwards.

The $0.1 \mathrm{~ns}$ increase in the lifetime value seems to be insignificant and is within the error limits, however, a similar raise (at the same pressure values) in the lifetime value for the second $o$-Ps component is observed also in a nitrogen atmosphere. This raise is not observed in pure nitrogen [9] and appears only with the co-presence of the resorcinol in the chamber. In this case, the lifetime value of the fourth component rises from $1 \mathrm{~ns}$ to $3 \mathrm{~ns}$.

The second $o$-Ps component in an argon atmosphere stabilizes at about $1 \mathrm{~ns}$ after reaching the said value at a gas pressure of $210 \mathrm{MPa}$ (similarly to the stabilization of the first described component).

Additional analyses using the MELT program [10] were performed to verify the obtained results. Due to the high requirements concerning the number of counts of the deconvoluted spectra, it was necessary to sum up the spectra collected under a similar gas pressure (pairs of spectra taken in the pressures of 90-120 MPa and 420-450 MPa). The levels of gas pressure used for the summing up were chosen specifically to gain information for both low and high pressure values.

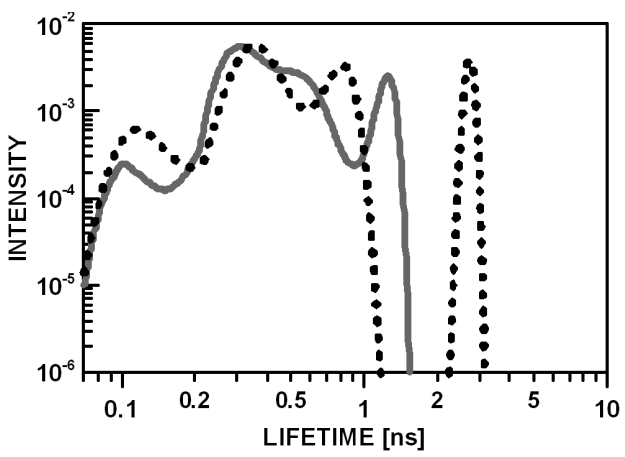

Fig. 5. MELT results (argon). Dashed line - 90$120 \mathrm{MPa}$; solid line - 420-450 MPa.

Figures 5 and 6 show the results obtained by the MELT analysis. In both cases the four components are clearly visible in the spectra at lower gas pressures disregarding which gas fills the chamber.

The previously described increase of the lifetime value of the fourth component, for the sample placed in the nitrogen-filled chamber, is also visible in the MELT analysis. When the chamber with the sample is filled with argon, the fourth component shortens and reaches values similar to those obtained by the LT program.

The third component shortens in both cases and it almost merges with the second component. When the

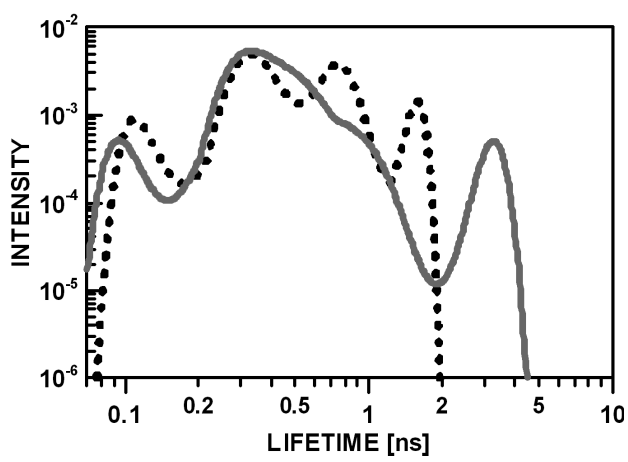

Fig. 6. MELT results (nitrogen). Dashed line - 90$120 \mathrm{MPa}$; solid line - 420-450 $\mathrm{MPa}$.

chamber with sample is filled with argon, the third component is still visible although it is not clearly separated from the component ascribed to free annihilation.

When nitrogen is used to apply pressure on the sample, one can see that the merge of the second and third components is almost complete. In fact, it appears that only one component with a broad distribution exists at this point. This of course can be affected by the previously mentioned spectra summing procedure, however, it stays consistent with the results obtained from the LT program.

The observed effect can be explained by the fact that the crystallographic structure of resorcinol is crushed in a gas pressure at about $200 \mathrm{MPa}$. This causes the free volumes to become accessible for the gas atoms, which can then fill them. Such a supposition can explain the merge of the second and the third component. The gas and the material create some sort of nanostructures. The gas mixes with the resorcinol molecules from the crushed crystal and the positrons seem to recognize this mixture as a single material which does not have a fully averaged electron density. Because nitrogen has the form of a two-atom molecule, which is significantly bigger than the argon molecule, it may form more complex structures, creating larger free volumes where positronium may be trapped, thus resulting in its longer lifetimes. One can also assume that the free volumes deform and as a result create new ones, which did not exist previously.

The second possible interpretation is that the phase transition occurs under a high pressure of gas (resorcinol exists in two phases as mentioned before) and the instable material creates a new structure. However, this explanation seems to be less probable as the phase transition effect should also be visible when the material was placed in an argon atmosphere.

\section{Conclusions}

The investigated material was placed into two different gas atmospheres (argon, nitrogen) and put under high pressure. Argon and nitrogen at high pressure penetrate the material, potentially causing a deformation of its primitive cell at higher pressure levels. 
Because of a difference in the molecular structure of the used gases, it is possible that new, bigger free volumes are formed during the interaction with nitrogen molecules. The gas and the material form a kind of nanostructure at higher gas pressures, which can be deduced from the broad distribution of the $\tau_{2}$ and $\tau_{3}$ values.

Such a phenomenon is possibly the reason why the $o$-Ps lifetime values rise with pressure over $210 \mathrm{MPa}$ when nitrogen is present in the chamber.

\section{References}

[1] Principles and Applications of Positron and Positronium Chemistry, Eds.: Y.C. Jean, E. Mallon, D.M. Schrader, World Sci., Singapore 2003.

[2] S.J. Tao, J. Chem. Phys. 56, 5499 (1972).

[3] M. Eldrup, D. Lightbody, J.N. Sherwood, Chem. Phys. 63, 51 (1981).
[4] T. Goworek, B. Jasińska, J. Wawryszczuk, K. Ciesielski, J. Chem. Soc. Faraday Trans. 92, 1573 (1996).

[5] B. Jasińska, A.E. Kozioł, T. Goworek, J. Radioanal. Nucl. Chem. 210, 617 (1996).

[6] J. Kansy, Nucl. Instrum. Methods Phys. Res. A 374, 235 (1996).

[7] J.C. Slater, J. Chem. Phys. 41, 3199 (1964).

[8] M. Tydda, B. Jasińska, A. Pieniążek, Nukleonika 58, 195 (2013).

[9] T. Goworek, J. Wawryszczuk, R. Zaleski, Chem. Phys. Lett. 402, 367 (2005).

[10] A. Shukla, M. Peter, L. Hoffmann, Nucl. Instrum. Methods Phys. Res. A 335, 310 (1993). 